\title{
Astronomical Significance of the 137.035 999 Part of the Inverse Fine-Structure Constant
}

\author{
Mels Sluyser ${ }^{1}$ \\ ${ }^{1}$ Driftweg 235, 1272 AD Huizen, The Netherlands \\ Correspondence: Mels Sluyser, Driftweg 235, 1272 AD Huizen, The Netherlands. E-mail: m.sluyser@planet.nl \\ Received: January 1, 2020 \\ Accepted: March 22, 2020 \\ Online Published: April 3, 2020 \\ doi:10.5539/apr.v12n2p42 \\ URL: http://dx.doi.org/10.5539/apr.v12n2p42
}

\begin{abstract}
The inverse fine-structure constant $1 / \alpha=137.035$ satisfies $1 / \alpha=11^{2}+4^{2}+0.035=121+16+0.035=137.035$, with 11 being the 11 dimensions of M-theory, 4 the number of dimensions of Einstein's space-time, and 0.035 the 3.5 percent visible Universe. Cosmological information appears to be encrypted linearly as a triplet code in $1 / \alpha$.
\end{abstract}

Keywords: Fine-structure constant -137

\section{Spatial Dimensions and 1/a}

The commonly used abbreviated expression in physics literature for the fine-structure constant alpha $(\alpha)$ is $\alpha=e^{2} / \hbar c$, where $e$ is the elementary charge, $\hbar$ the reduced Planck constant, and $c$ the speed of light in vacuum.

Since the early 1900s, physicists have suspected that the number 137 in the inverse fine-structure constant $1 / \alpha=$ $137.035999 \ldots$ would lay at the heart of a grand unified theory, relating theories of electromagnetism, quantum mechanics and gravity. However, thus far no reasonable theory was presented. Richard Feynman, one of the top investigators in this field even suggested that all physicists should put a sign in their offices with the number 137, to remind them how much they did not know (Feynman, 1985).

A few years ago it was proposed that the 137 should be read as 4 squared plus 11 squared, and that these numbers 4 and 11 relate respectively to the 4 dimensions of Einstein's space-time, and the 11 dimensions of M-theory (Sluyser, 2016). Now, to add to this idea it is here suggested that the 035 triplet in $1 / \alpha$, by standing for 0.035 means a 3.5 percent visible universe, thereby indicating 96.5 percent dark energy and dark matter. The smallest uncertainty regarding alpha comes from comparing the theoretical expression and experimental value of the anomalous magnetic moment of the electron, yielding as a value 1/ $\alpha=137.035999084$ (21) as reported by CODATA, NIST (Mohr et al., 2019). However, starting in the 1980's a new and wholly different measurement approach using the quantum Hall effect (QHE) has caused interest, because the value of alpha $(\alpha)$ obtained from it independently corroborates the value of alpha from the electron magnetic moment anomaly (Cage et al., 2012; Aoyama et al., 2012).

The QHE value of alpha does not have as little uncertainty as the electron magnetic moment value, but it does provide with $1 / \alpha=137.035999173(35)$ a significant independent confirmation of that value. Interestingly, both

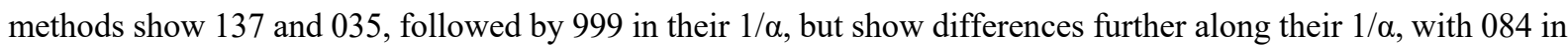
the CODATA and 173 in the QHE. This may indicate that the 137.035 part of the fine-structure constant exerts a specific key-like locking function, with the subsequent 999 triplet perhaps acting as a 'stop codon', by blocking further action along the $1 / \alpha$. 
It should be pointed out that generally the percentage visible Universe is estimated to be about 4 (Panek, 2011), so it will be interesting if a more accurate percentage will eventually turn out to be 3.5 , as used in this paper.

\section{References}

Aoyama, T. , Hayakawa, M. , Kinoshita, T. , \& Nio, M. . (2012). Tenth-order qed contribution to the electron g - 2 and an improved value of the fine structure constant. Physical Review Letters, 109(11), p.111807.1-111807.4.

Cage, M.E. et al. (2012). The Quantum Hall Effect. Berlin: Springer.

Feynman, R. P. (1985) QED: The Strange Theory of Light and Matter. Princeton: Princeton University Press, p.129.

Mohr, P. J., Taylor, B. N., \& Newell, D. B. (2019). Fine structure constant CODATA. Internationally recommended 2018 values of the fundamental physical constants. National Institute of Standards and Technology.

Panek, R. (2011). The 4 Percent Universe. Dark Matter, Dark Energy, and the Race to discover the Rest of Reality. Houghton Mufflin Harcourt.

Sluyser, M. (2016). Speculation on the Number 137 in the Fine-Structure Constant. Applied Physics Research, $8(3), 58-59$.

\section{Copyrights}

Copyright for this article is retained by the author(s), with first publication rights granted to the journal.

This is an open-access article distributed under the terms and conditions of the Creative Commons Attribution license (http://creativecommons.org/licenses/by/4.0/). 